Check for updates

Cite this: RSC Adv., 2017, 7, 32193

Received 5th May 2017

Accepted 18th June 2017

DOI: $10.1039 / \mathrm{c} 7 \mathrm{ra05095j}$

rsc.li/rsc-advances

\section{Root-like natural fibers in polypropylene prepared via directed diffusion and self-assembly driven by hydrogen bonding}

\begin{abstract}
Pei Huang, ${ }^{a}$ Shaohong Shi, ${ }^{a}$ Yuansen Liu, ${ }^{b}$ Min Nie (D) *a and Qi Wang ${ }^{a}$
Interfacial properties govern effective transfer of load from a polymer matrix to a reinforced fiber, dictating the mechanical performance of the composite. This paper reports a facile and controlled strategy of preparing root-like natural fibers (NFs) in polypropylene (PP), where the two components are integrated via mechanical interlocking provided by the interfacial grown fiber. Specifically, driven by hydrogen bonding between amide groups of the self-assembling molecules (NAs) and abundant hydroxyl groups existing in the NF, the former selectively diffuses and aggregates on the latter and then self-assembles into branched fibers, which can be tailored finely by the annealing temperature and time as well as cooling rate. This study opens up an interfacial manipulation without preliminary surface modification and holds great potential in preparing NF-reinforced polymer composites.
\end{abstract}

\section{Introduction}

Polymer composites have comprehensive properties superior to the parent polymer and thus attract significant attention in the field of materials science and industrial applications. ${ }^{1,2}$ The inherent incompatibility between most polymers and introduced fillers results in poor interfacial interaction and finally inferior mechanical performances. ${ }^{3}$ Currently, common ways to improve the interfacial interaction include modifying the filler surface and adding some compatibilizers to minimize the interfacial tension. However, there are still some issues that need to be addressed for polymer composites, such as high consumption of toxic reagents, the soft nature of the lowmolecular weight compatibilizer, and so on., ${ }^{4,5}$

It's evident that interfacial geometry of inorganic filler can influence the interfacial interaction of polymer composites. ${ }^{6}$ When the fillers are mechanically interlocked with polymer matrix, large interfacial friction is generated to promote interfacial load-transferring efficiency and to perfect the resulting composite..$^{7-10}$ Tamrakar achieved the controlled growth of carbon nanotubes at the surface of glass fibers (GF) by means of electrophoretic deposition to significantly enhance the interfacial strength between the fiber and epoxy resin. ${ }^{8}$ Our group also utilized the polarity gaps of the different components to direct interfacial diffusion and recrystallization of some amide-based

\footnotetext{
${ }^{a}$ State Key Laboratory of Polymer Materials Engineering, Polymer Research Institute of Sichuan University, Chengdu 610065, China. E-mail: poly.nie@gmail.com; Fax: +8628-85405133; Tel: +86-28-85405133

${ }^{b}$ Engineering Research Center of Marine Biological Resource Comprehensive Utilization, Third Institute of Oceanography, State Oceanic Administration, Xiamen, 361005, China
}

self-assembling compounds and prepared pompon-like hydroxyapatite and root-like glass fiber, where the grown fibers at the interface served as interlocks to tightly join the two components. ${ }^{11,12}$ However, the self-assembling molecules generally present high polarity so pristine fillers can't effectively anchor and immobilize the molecules. Accordingly, a polydopamine layer with hydroxyl groups needs to be pre-coated onto the surface for the directed interfacial diffusion.

Benefited from the renewability, abundant availability, lightweight and excellent mechanical properties, natural fibers (NFs) can replace traditional fillers as a green and high-efficient reinforcement for polymer and is becoming a popular choice for preparation of polymer composites. ${ }^{13,14}$ A prominent feature of NFs is rich hydroxyl groups at the surface so they have the potential to function as a preferred acceptor to assemble the amide-based molecules via hydrogen bonding. ${ }^{15}$ Accordingly, in this paper, an amide-based compound (WBG) which dissolves into polypropylene (PP) melts at high temperature and selfassembles upon cooling, ${ }^{16-18}$ was chosen and the potential interaction with NFs was explored. Then, the interfacial nucleation and growth were investigated and the corresponding regulation mechanism was proposed. Finally, root-like NFs with controlled branched density and dimension were in situ built in PP melts.

\section{Experiment}

\section{Materials}

Isotactic polypropylene (trade name: T30S) with weight average molecular weight of $400 \mathrm{~kg} \mathrm{~mol}^{-1}$ and the molecular weight distribution of 4.6, was provided by Dushanzi Petroleum Chemical Incorporation (Xinjiang, China). Rare earth 
compound (trade name: WBG-II) was supplied by Guangdong Weilinna Incorporation (Guangdong, China). It's a kind of dimetal complex of lanthanum and calcium with dicarboxylic acid and amide-type ligands and the general formula is $\mathrm{Ca}_{x^{-}}$ $\mathrm{La}_{1-x}(\mathrm{LIG} 1)_{m}(\mathrm{LIG} 2)_{n}$, where $x$ and $1-x$ are corresponding to the proportion of $\mathrm{Ca}^{2+}$ and $\mathrm{La}^{3+}$ ion in the complex, and LIG1 and LIG2 represent dicarboxylic acid and amide-type ligand with coordination numbers of $m$ and $n .{ }^{19}$ The average size is $\sim 20 \mathrm{~nm}$. Cotton fiber was offered by Shanghai Old Cotton Yarn Company (Shanghai, China) and was extracted by dimethylsulfoxide to remove the impurities. The diameter is $\sim 15 \mu \mathrm{m}$.

\section{Sample preparation}

PP samples containing $0.3 \mathrm{wt} \%$ of rare earth compounds (NAs) were prepared in a co-rotating twin screw extruder at the temperature of $180{ }^{\circ} \mathrm{C}$ and hot-pressed into $\sim 10 \mu \mathrm{m}$ thin films. Then, a single cotton fiber was embedded between the two PP with NAs films to prepare the composites.

\section{Characterization}

Fourier transform infrared spectroscopy (FTIR). NF and NA powders were mixed at the weight ratio of $1: 1$ and the FTIR spectra were recorded from 4000 to $600 \mathrm{~cm}^{-1}$ on a Nicolet 20SXB spectrometer (Thermo Fisher Scientific Inc., USA). As a control, pristine NF and NA powders were also measured.

Polarized light microscope (PLM) observation. The assembly that single fiber was sandwiched between the two NAscontaining PP film, was placed under PLM (Leica DM2500P) connected with hot stage (Linkam THMS600, Linkam Scientific Instruments Ltd., UK), heated to a given temperature and kept for a certain time, followed by cooling down at a constant rate to the preset temperatures. The $\mathrm{PP} / \mathrm{NF}$ interfacial structures were recorded.

Scanning electron microscope (SEM) investigation. $\sim 5 \mathrm{~g}$ NAs were dissolved into $100 \mathrm{ml}$ 1,2-dichlorobenzene. Then, NFs were put into the NA solution for $0.5 \mathrm{~h}$ at $80{ }^{\circ} \mathrm{C}$. The surface morphology of the treated NFs was investigated by a FEI Inspect F-SEM instrument with an acceleration voltage of $20 \mathrm{kV}$.

\section{Results and discussion}

Hydroxyl groups enriched at the surface of NF can provide the possibility for the formation of hydrogen bonds with amide group carried by NAs. The underlying interaction between NA and NF was evaluated by FTIR spectra (Fig. 1a). NF exhibited the broad peaks at $3432 \mathrm{~cm}^{-1}$ due to the presence of -OH groups; the sharp absorption at $3317 \mathrm{~cm}^{-1}$ with a shoulder peak appeared in the NAs, where the former was corresponding to self-association $-\mathrm{NH}$ groups and the latter came from free ones. $^{20}$ One can observe that in the case of NF/NA mixture, the shoulder representing free - $\mathrm{NH}$ groups took the distinct shift from $3458 \mathrm{~cm}^{-1}$ to $3421 \mathrm{~cm}^{-1}$, accompanied by a blue shift of $-\mathrm{C}=\mathrm{O}$ group situated at $1718 \mathrm{~cm}^{-1}$. These features confirmed that there was hydrogen bonding between amide groups of the NAs and abundant hydroxyl groups existing in the NF. This was also verified by the coating of NF by some NAs via dip-coating experiment, as shown in Fig. 1b. Similar phenomena were also investigated by previous researchers. ${ }^{21}$ As melting PP is nonpolar, the existence of NF-NA hydrogen bonding guided the directed diffusion of NA onto the surface of NF and triggered the subsequent epitaxial crystallization upon cooling, into root-like fiber with laterally grown NA fiber. The corresponding mechanism on the formation of root-like NF in PP matrix is proposed, as illustrated in Fig. 1c.

For convenient observation, a single NF was embedded in two PP films with $0.3 \mathrm{wt} \%$ NAs and heated to $250^{\circ} \mathrm{C}$, where NAs completely dissolved into PP melt. ${ }^{12}$ One can observe that upon cooling, the dissolved NAs gradually separated out from PP melts to crystallize epitaxially on the NF surface. As shown in Fig. 2b-e, in the initial stage, only few short NAs fibers of $13 \mu \mathrm{m}$ emerged on the surface of NF; with the decreasing temperature, the density and dimension of the interracially-grown NAs fibers increased. When the temperature reached $156{ }^{\circ} \mathrm{C}$, the NAs fibers grew to $90 \mu \mathrm{m}$ and completely covered the NF. Moreover, ascribing to the highly nucleating efficiency of NA on $\mathrm{PP},{ }^{22}$ the branched NA fibers held rich heterogeneous nuclei for PP and induced the lateral growth of PP crystals, into transcrystalline layers featuring the excellent interfacial interaction (Fig. 2f). Analogs to tree root, the branched NA fiber can interlock NF
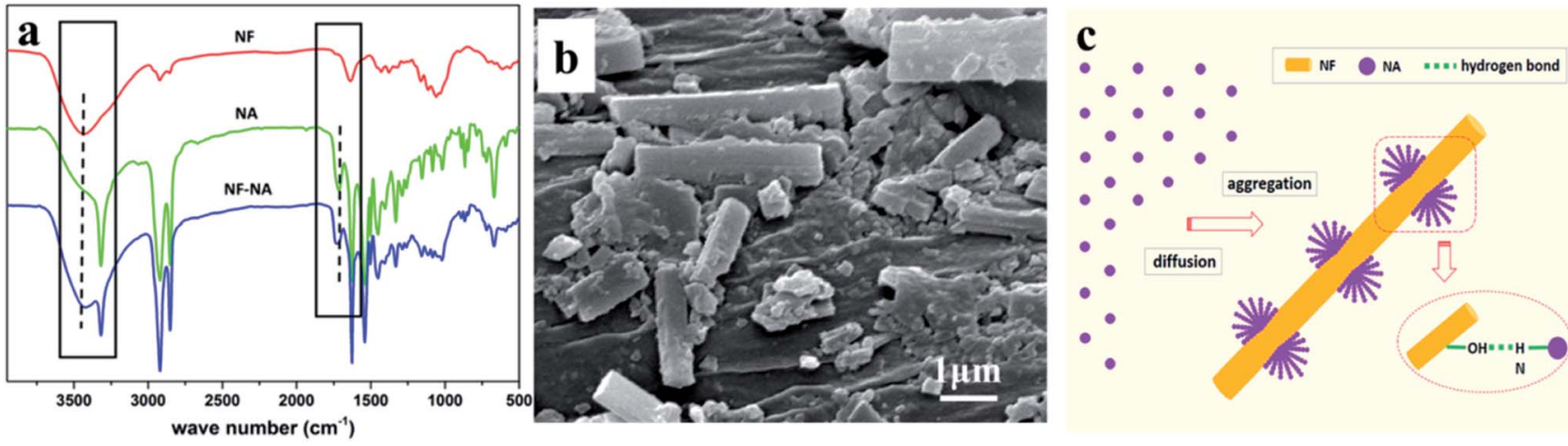

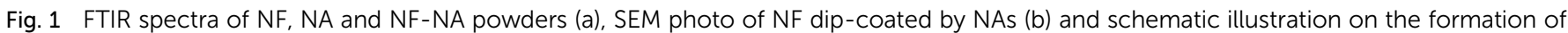
root-like NF in PP matrix (c). 

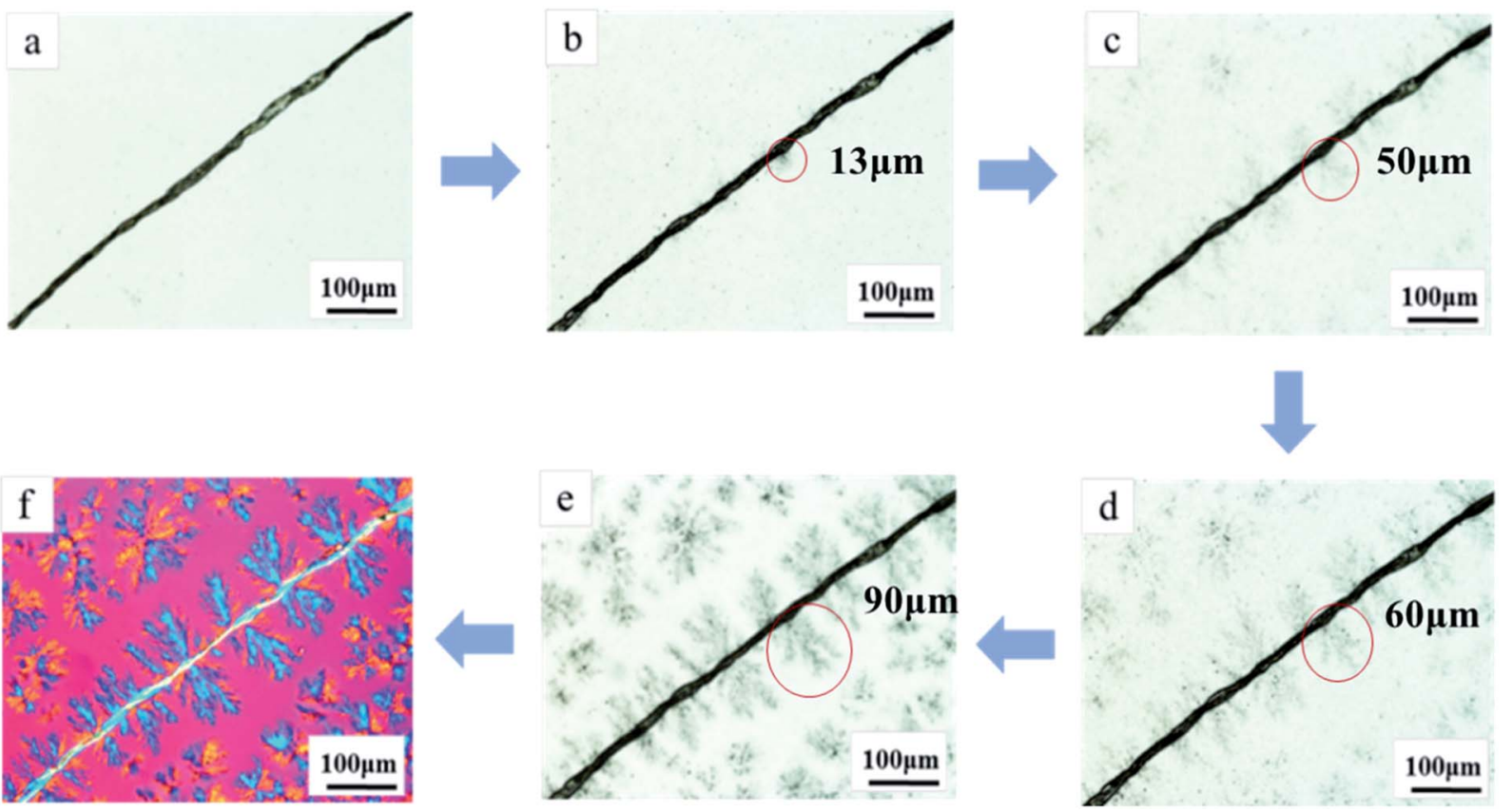

Fig. 2 PLM photos for NF/PP containing 0.3 wt $\%$ NA annealed at $250{ }^{\circ} \mathrm{C}$ for 2 min during the cooling process at a rate of $30{ }^{\circ} \mathrm{C}$ min ${ }^{-1}$ : (a) $250{ }^{\circ} \mathrm{C}$; (b) $170{ }^{\circ} \mathrm{C}$; (c) $162{ }^{\circ} \mathrm{C}$; (d) $156{ }^{\circ} \mathrm{C}$; (e) $140{ }^{\circ} \mathrm{C}$; (f) the corresponding photo of (e) taken in polarized mode.

with surrounding PP matrix to significantly enhance the interfacial friction, resulting in the difficulty of NF pulling-out from polymer matrix under mechanical load. Accordingly, the load can transfer effectively from PP matrix to the NF through the interfacially-grown NA fiber, which in turn endowed the strong interfacial interaction of the NF-PP interface. ${ }^{9,10}$ Different from the previous chemical modification methods, this study provided a new idea to modify the interface between the fillers and polymer matrix via interfacial interlocking; moreover, compared to the glass fiber or hydroxyapatite featuring inert activity to NAs, NFs carry massive active hydroxyl groups to interact with amide groups of NA so the interfacial fibrous crystals can be directly generated without preliminary surface modification.

The formation process of root-like NFs involved the directed diffusion of the NA molecules at the NF surface and the subsequent epitaxial growth. Therefore, fine control of the interfacial grown NA fibers can be achieved by adjusting the diffusion and growth processes. The molecular diffusion is a kinetic process, depending on the annealing temperature and time. As shown in Fig. 3a-c, with the increasing annealing temperature, NAs displayed stronger mobility and the interfacial diffusion became more remarkable, leading to denser and longer branched NA fibers. Further, at the prolonged annealing time, NAs had higher
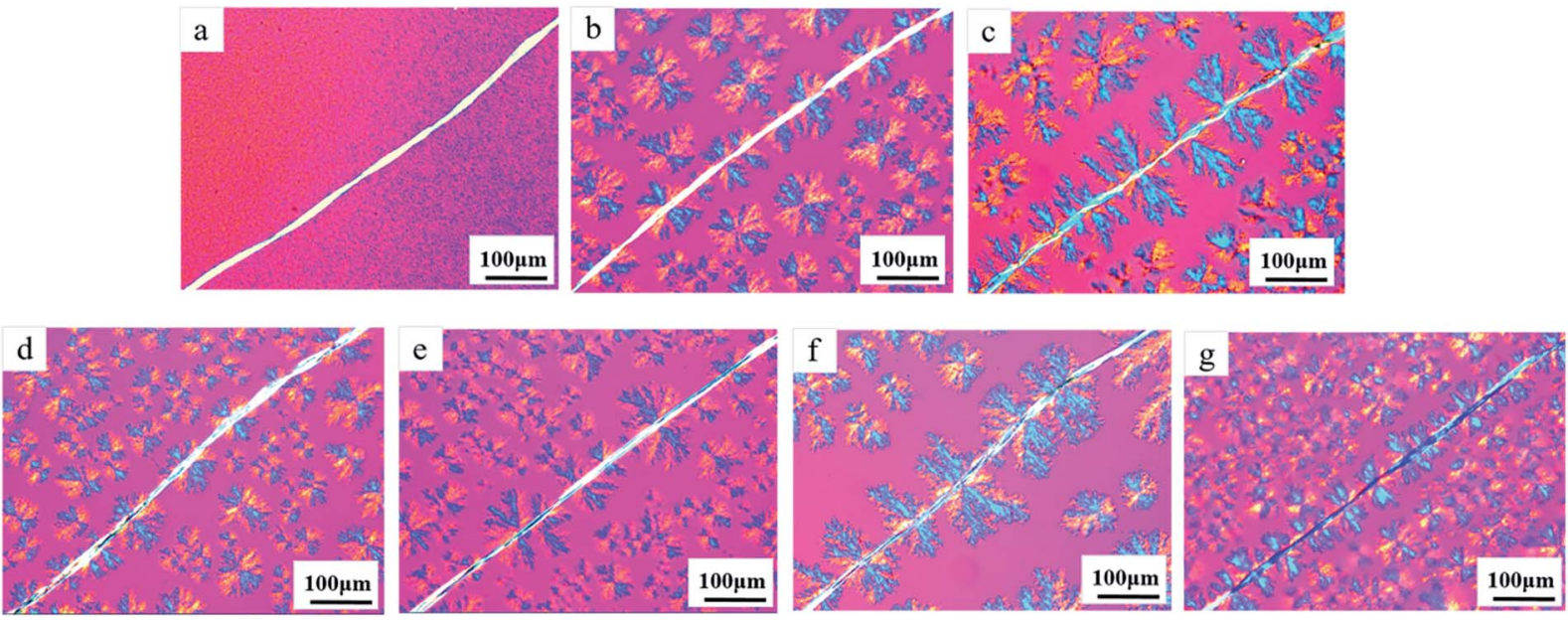

Fig. 3 PLM photos for single NF/PP with $0.3 \%$ NA annealed for 2 min at different temperatures followed by cooled at the rate of $30{ }^{\circ} \mathrm{C}$ min ${ }^{-1}$. $200^{\circ} \mathrm{C}$ (a), $230^{\circ} \mathrm{C}$ (b) and $250^{\circ} \mathrm{C}$ (c); cooled at the rate of $30^{\circ} \mathrm{C} \mathrm{min}^{-1}$ annealed at $230^{\circ} \mathrm{C}$ for different annealing times: $0.5 \mathrm{~min}$ (d) and $1 \mathrm{~min}$ (e); annealed for $2 \mathrm{~min}$ at $230^{\circ} \mathrm{C}$ followed by cooled at different cooling rate: $10^{\circ} \mathrm{C} \mathrm{min}^{-1}$ (f) and $50{ }^{\circ} \mathrm{C} \mathrm{min}{ }^{-1}$ (g). 
probability to diffuse from PP bulk matrix to the NF surface and thus root-like NF with larger and denser NA fibers was generated. Additionally, the growth of branched NA fiber can be regulated by controlling the cooling rate. Slow cooling can facilitate the perfect growth of NA fibers. As shown in Fig. $3 f$ and $g$, when the cooling rate was reduced from 50 to $10{ }^{\circ} \mathrm{C} \mathrm{min}^{-1}$, larger NA fibers were generated at the surface of NF.

\section{Conclusions}

The hydrogen bonding between amide groups of NAs and abundant hydroxyl groups existing in the NF fibers can direct the interfacial diffusion of the former and induce the lateral growth at the NF surface, into root-like NFs with mechanically interlocking with PP matrix, which is favorable to the load transferring from polymer matrix to the reinforced NF. Moreover, fine control of the interfacial grown NA fibers is achieved by adjusting the diffusion and growth processes, which are in turn determined by the annealing temperature, annealing time and cooling rate. Compared to conventional chemical modification, the functional interfacial geometry is in situ formed in PP melts without any complicated chemical pre-treatment, which holds great potential in the NF-reinforced polymer composites.

\section{Acknowledgements}

This work is financiered by State Key Laboratory of Polymer Materials Engineering (Grant No. sklpme2016-3-05), the Engineering Research Center of Marine Bioresources Comprehensive Utilization, SOA, (MBRCU201601), the National Natural Science Foundation of China (51421061), the Program of Innovative Research Team for Young Scientists of Sichuan Province (2016TD0010) and the Program of Introducing Talents of Discipline to Universities (B13040).

\section{References}

1 F. Khelifa, Y. Habibi, L. Bonnaud and P. Dubois, Epoxy monomers cured by high cellulosic nanocrystal loading, ACS Appl. Mater. Interfaces, 2016, 8, 10535-10544.

2 C. Baley, A. Kervoëlen, A. Le Duigou, C. Goudenhooft and A. Bourmaud, Is the low shear modulus of flax fibres an advantage for polymer reinforcement, Mater. Lett., 2016, 185, 534-536.

3 N. Y. Ning, S. R. Fu, W. Zhang, F. Chen, K. Wang, H. Deng, Q. Zhang and Q. Fu, Realizing the enhancement of interfacial interaction in semicrystalline polymer/filler composites via interfacial crystallization, Prog. Polym. Sci., 2012, 37, 1425-1455.

4 N. P. Panapitiya, S. N. Wijenayake, D. D. Nguyen, Y. Huang, I. H. Musselman, K. J. Balkus and J. P. Ferraris, Gas separation membranes derived from high-performance immiscible polymer blends compatibilized with small molecules, ACS Appl. Mater. Interfaces, 2015, 7, 18618-18627.

5 L. Xie, B. Shan, X. Sun, Y. Tian, H. Xie, M. He, Y. Xiong and Q. Zheng, Natural fiber-anchored few-layer graphene oxide nanosheets for ultrastrong interfaces in poly(lactic acid), ACS Sustainable Chem. Eng., 2017, 5, 3279-3289.

$6 \mathrm{~K}$. Kendall, The adhesion and surface energy of elastic solids, J. Phys. D: Appl. Phys., 1971, 4, 1186.

7 X. Jin, J. Strueben, L. Heepe, A. Kovalev, Y. K. Mishra, R. Adelung, S. N. Gorb and A. Staubitz, Joining the unjoinable: adhesion between low surface energy polymers using tetrapodal ZnO linkers, Adv. Mater., 2012, 24, 56765680 .

8 S. Tamrakar, Q. An, E. T. Thostenson, A. N. Rider, B. Z. Haque and J. W. Gillespie, Tailoring interfacial properties by controlling carbon nanotube coating thickness on glass fibers using electrophoretic deposition, ACS Appl. Mater. Interfaces, 2016, 8, 1501-1510.

9 C. Cui, W. Qian, M. Zhao, F. Ding, X. Jia and F. Wei, High strength composites using interlocking carbon nanotubes in a polyimide matrix, Carbon, 2013, 60, 102-108.

10 W. S. Kim, I. H. Yun, J. J. Lee and H. T. Jung, Evaluation of mechanical interlock effect on adhesion strength of polymer-metal interfaces using micro-patterned surface topography, Int. J. Adhes. Adhes., 2010, 30, 408-417.

11 X. He, Y. Li, M. Nie and Q. Wang, Root-like glass fiber with branched fiber prepared via molecular self-assembly, RSC Adv., 2016, 6, 45492-45494.

$12 \mathrm{X}$. He, W. Xu, Y. Liu, M. Nie and Q. Wang, In situ construction of pompon-like hydroxyapatite hybrid via interfacial self-assembly in polypropylene matrix, Compos. Sci. Technol., 2017, 142, 246-252.

13 S. K. Ramamoorthy, M. Skrifvars and A. Persson, A review of natural fibers used in biocomposites: plant, animal and regenerated cellulose fibers, Polym. Rev., 2015, 55, 107-162.

14 O. Faruk, A. K. Bledzki, H. P. Fink and M. Sain, Biocomposites reinforced with natural fibers: 2000-2010, Prog. Polym. Sci., 2012, 37, 1552-1596.

15 G. W. Wang, B. Chen, L. H. Zhuang, K. Yun, J. R. Guo, Y. Wang and B. Xu, Dyeing performances of ramie fabrics modified with an amino-terminated aliphatic hyperbranched polymer, Cellulose, 2015, 22, 1401-1414.

16 F. Luo, C. Geng, K. Wang, H. Deng, F. Chen, Q. Fu and B. Na, New understanding in tuning toughness of $\beta$-polypropylene: the role of $\beta$-nucleated crystalline morphology, Macromolecules, 2009, 42, 9325-9331.

17 X. Q. Hao, G. Q. Zheng, K. Dai, Z. H. Jia, Q. Jia, J. B. Chen, C. T. Liu and C. Y. Shen, Facile preparation of rich betatranscrystallinity in PET fiber/iPP composites, eXPRESS Polym. Lett., 2011, 5, 1017-1026.

18 C. Zhang, B. Wang, J. Yang, D. Ding, X. Yan, G. Zheng, K. Dai, C. Liu and Z. Guo, Synergies among the self-assembled $\beta$ nucleating agent and the sheared isotactic polypropylene matrix, Polymer, 2015, 60, 40-49.

19 W. Xiao, P. Wu and J. Feng, Effect of $\beta$-nucleating agents on crystallization and melting behavior of isotactic polypropylene, J. Appl. Polym. Sci., 2008, 108, 3370-3379.

20 R. S. Shih, C. Lu, H. S. W. Kuo and F. C. Chang, Hydrogen bond-mediated self-assembly of polyhedral oligomeric silsesquioxane-based supramolecules, J. Phys. Chem. C, 2010, 114, 12855-12862. 
21 Y. Xin, X. Kong, X. Zhang, Z. Lv and X. Du, Self-assembly and molecular recognition of adenine- and thyminefunctionalized nucleolipids in the mixed monolayers and thymine-functionalized nucleolipids on aqueous melamine at the air-water interface, Langmuir, 2012, 28, 11153-11163.
22 X. Wen, Y. Li, M. Nie, Q. Wang and X. Zhang, Formation mechanism of hybrid shish-kebab and its reinforcing effects on polypropylene, Polym.-Plast. Technol. Eng., 2016, 55, 775-783. 